F. ICHIKAWA

KODAI MATH. J.

15 (1992), 134-140

\title{
THOM'S CONJECTURE ON SINGULARITIES OF GRADIENT VECTOR FIELDS
}

\author{
By Fumio ICHIKAWA
}

\section{Introduction.}

In [3], R. Thom gave the following conjecture.

CONJECTURE. Let $f(x)$ be a germ of real analytic function at the origin $0 \in R^{n}$ and let $X=\operatorname{grad} f(x)$ be the gradient vector field of $f(x)$ with respect to the ordinary Riemannian metric on $R^{n}$. If an integral curve $g(t)$ of $X$ tends to the origin $0 \in R^{n}$, then there exists a unique tangential direction $\lim _{t \rightarrow+\infty} g(t) /|g(t)|$.

Thom proved the case where $f(x)$ is a homogeneous polynomial and for the general case he gave an outline of a proof. In this paper, we give a partial answer to the above problem. The essential idea of our proof is the same as Thom's one (see [3]).

Let $f(x):\left(R^{n}, 0\right) \rightarrow(R, 0)$ be a germ of analytic function. And we express $f(x)$ in the form

$$
f(x)=P_{k}(x)+P_{k+1}(x)+\cdots+P_{m}(x)+\cdots
$$

where $P_{m}(x)$ is a homogeneous polynomial of degree $m$.

We define the cone spectrum $S p\left(P_{m}\right)$ as follows:

$$
S p\left(P_{m}\right)=\left\{x=\left(x_{1}, \cdots, x_{n}\right) \in R^{n} ; x_{i} \frac{\partial P_{m}}{\partial x_{\jmath}}=x_{j} \frac{\partial P_{m}}{\partial x_{\imath}} i, j=1, \cdots, n\right\} .
$$

Obviously, $S p\left(P_{m}\right)$ is a cone algebraic set and it contains $0 \in R^{n}$.

In this paper, we prove the following theorem.

THEOREM. Let $f(x)=P_{k}(x)+P_{k+1}(x)+\cdots$ be a real analytic function germ at $0 \in R^{n}$. If $\operatorname{dim} S p\left(P_{k}\right) \leqq 1$, then any integral curve of $\operatorname{grad} f(x)$ which tends to $0 \in R^{n}$ has a unique tangential direction at the origin.

Remark. We see later on that the condition $\operatorname{dim} S p\left(P_{k}\right) \leqq 1$ is equivalent to that the restricted function $\left.P_{k}\right|_{s^{n-1}}$ of $P_{k}(x)$ to the unit sphere $S^{n-1}$ has only isolated singularities. Thus, the above condition is a generic property on the initial term of $f(x)$.

Received June 21, 1991. 
COROLlARY. In the two dimensional case, Thom's conjecture holds.

\section{Lojasiewicz's Theorem and Blowing up of vector field.}

The proof of our theorem is based on two important theorems. One is Lojasiewicz's theorem on analytic gradient vector fields and the other is Takens's blowing up construction of singularities of vector fields.

Now, for its importance we start with recalling them.

LEMMA 2.1. Let $f(x)$ be a real analytic function defined on a neighbourhood $U$ of $a \in R^{n}$ and $f(a)=0$. Then, there exists $0<\theta<1$ such that

$$
|\operatorname{grad} f(x)| \geqq|f(x)|^{\theta}
$$

in some neibourhood of $a \in R^{n}$.

The proof can be found in Lojasiewicz [1] pp 92 .

THEOREM 2.2. (Lojasiewicz) Let $f(x)$ be a real analytic function defined on a neibourhood $U$ of $R^{n}$ and let $A$ denote the set $f^{-1}(0) \cap U$. If an integral curve $g(t)$ of $\operatorname{grad} f(x)$ tends toward $A$, then $g(t)$ tends to a unique point of $A$.

Proof. Let $g(t)=\left(g_{1}(t), g_{2}(t), \cdots, g_{n}(t)\right)$ denote the integral curve of $\operatorname{grad} f(x)$ with $g(0)=x, x \in U$ i.e.

$$
\frac{d g}{d t}(t)=\left(\frac{\partial f}{\partial x_{1}}(g(t)), \cdots, \frac{\partial f}{\partial x_{n}}(g(t))\right) \text { and } g(0)=x .
$$

Now, easily we have

$$
\begin{aligned}
\frac{d}{d t} f(g(t)) & =\frac{\partial f}{\partial x_{1}} \frac{\partial g_{1}}{\partial t}+\cdots+\frac{\partial f}{\partial x_{n}} \frac{\partial g_{n}}{\partial t} \\
& =|\operatorname{grad} f(g(t))|^{2} \geqq 0 .
\end{aligned}
$$

On the other hand. if $g(t)$ tends to a point $a \in A$, then from Lemma 2.1, there exists $0<\theta<1$ such that

$$
|\operatorname{grad} f(x)| \geqq|f(x)|^{\theta}
$$

in some neibourhood of $a \in U$.

Then, the length of integral curve $g(t)$ from $t=0$ to $t=m$ is estimated as follows :

$$
\begin{aligned}
\int_{0}^{m}|\operatorname{grad} f(g(t))| d t & =\int_{0}^{m} \frac{(d / d t) f(g(t))}{|\operatorname{grad} f(g(t))|} d t \\
& \leqq \int_{0}^{m} \frac{(d / d t) f(g(t))}{|f(g(t))|^{\theta}} d t
\end{aligned}
$$




$$
\begin{aligned}
& =\frac{-1}{1-\theta} \int_{0}^{m} \frac{d}{d t}\left[\left(-f(g(t))^{1-\theta}\right] d t\right. \\
& =\frac{1}{1-\theta}\left[(-f(x))^{1-\theta}-\left(-f(g(m))^{1-\theta}\right]\right. \\
& <\frac{1}{1-\theta}(-f(x))^{1-\theta}<\infty .
\end{aligned}
$$

Here, from (1) we note that $f(g(t))$ is an increasing function and $\lim _{t \rightarrow+\infty} f(g(t))$ $=f(a)=0$, thus $f(g(t))<0$. If the $\omega$-limit set of $g(t)$ contains two or more points, then the length of integral curve $g(t)$ must be $\infty$. This contradicts the above estimation.

From Theorem 2.2, we can easily obtain the following.

Proposition 2.3. Let $M^{n}$ be a real analytic Riemannian manifold of dimension $n$ and $f: M^{n} \rightarrow R$ be a real analytic function on $M^{n}$. Then, every integral curve $g(t)$ of $\operatorname{grad} f(x)$ has unique $\alpha$-limit and $\omega$-limit points. Moreover, the points $\lim _{t \rightarrow+\infty} g(t)$ and $\lim _{t \rightarrow-\infty} g(t)$ are singular points of $f(x)$.

Next, we recall the blowing-up construction of vector field. For more details see Takens [2].

Theorem 2.4. (Takens [2]) Let $X$ be a $C^{\infty}$-vector field on $R^{n}$ with $X(0)=0$. Let $\Phi: S^{n-1} \times R \rightarrow R^{n}$ be a $C^{\infty}$-mapping defined by $\Phi\left(\bar{x}_{1}, \cdots, \bar{x}_{n}, r\right)=\left(r \bar{x}_{1}, \cdots, r \bar{x}_{n}\right)$ where $\left(\bar{x}_{1}, \cdots, \bar{x}_{n}\right)$ with $\sum_{\imath=1}^{n} \bar{x}_{2}^{2}=1$ is the coordinate system of $S^{n-1}$. Then there exists a $C^{\infty}$-vector field $\tilde{X}$ such that $\Phi_{*}(\tilde{X})=X$.

For the purpose of our proof, we repeat shortly the outline of Takens's proof.

Proof. From a direct calculation we have

$$
\left(\sum_{\imath=1}^{n} x_{\imath}^{2}\right) X=\langle R, X\rangle R+2 \sum_{\imath, j=1}^{n}\left\langle V_{\imath j}, X\right\rangle V_{\imath \jmath}
$$

where $R, V_{\imath \jmath}$ are the vector fields on $R^{n}$ given by

$$
R=\sum_{\imath=1}^{n} x_{i} \frac{\partial}{\partial x_{\imath}}, \quad V_{\imath \jmath}=\frac{1}{2}\left(x_{i} \frac{\partial}{\partial x_{\jmath}}-x_{j} \frac{\partial}{\partial x_{\imath}}\right)
$$

and $\langle$,$\rangle denotes the inner product of R^{n}$.

We define the vector fields $\tilde{R}$ and $\tilde{V}_{\imath}$ on $S^{n-1} \times R$ by

$$
\widetilde{R}=r \frac{\partial}{\partial r}, \quad \tilde{V}_{\imath \jmath}=\frac{1}{2}\left(\bar{x}_{i} \frac{\partial}{\partial \bar{x}_{j}}-\bar{x}_{j} \frac{\partial}{\partial \bar{x}_{\imath}}\right) \text {. }
$$

Then, we have

$$
\Phi_{*}(\widetilde{R})=R \quad \text { and } \quad \Phi_{*}\left(\tilde{V}_{\imath \jmath}\right)=V_{\imath \jmath}
$$

Now, it is clear that the vector field 


$$
\tilde{X}=\frac{1}{r^{2}}\left[(\langle R, X\rangle \circ \Phi) \tilde{R}+2 \sum_{\imath, \jmath=1}^{n}\left(\left\langle V_{\imath \jmath}, X\right\rangle \circ \Phi\right) \tilde{V}_{\imath \jmath}\right]
$$

satisfies the required condition $\Phi_{*}(\tilde{X})=X$.

Remark. If the $(k-1)$-jet of $X$ at the origin equals 0 , in other words the degree of initial term of $X$ is $k$, then we set

$$
\bar{X}=\frac{1}{r^{k+1}}\left[(\langle R, X\rangle \circ \Phi) \tilde{R}+2 \sum_{\imath, \jmath=1}^{n}\left(\left\langle V_{\imath \jmath}, X\right\rangle \circ \Phi\right) \tilde{V}_{\imath \jmath}\right] .
$$

Then $\bar{X}$ is also $C^{\infty}$-vector field on $S^{n-1} \times R$ and the integral curves of $\tilde{X}$ and $\bar{X}$ coincide as sets. Thus the $\omega$-limit sets of $\tilde{X}$ and $\bar{X}$ coincide. We say that $\bar{X}$ is the blowing-up of $X$ at $0 \in R^{n}$.

\section{The proof of theorem.}

Now, let $f(x)=P_{k}+P_{k+1}+\cdots:\left(R^{n}, 0\right) \rightarrow(R, 0)$ be a real analytic function germ. Then the blowing-up vector field $\bar{X}$ of $\operatorname{grad} f(x)$ is given by:

$$
\left.\bar{X}=\frac{1}{r^{k}}[\langle R, \operatorname{grad} f(x)\rangle \circ \Phi) \tilde{R}+2 \sum_{\imath, \jmath=1}^{n}\left(\left\langle V_{\imath \jmath}, \operatorname{grad} f(x)\right\rangle \circ \Phi\right) \tilde{V}_{\imath \jmath}\right] .
$$

Then the restriction of vector field $\bar{X}$ to $S^{n-1} \times\{0\}$ is given by

$$
2 \sum_{\imath, j=1}^{n}\left\langle V_{\imath \jmath}, \operatorname{grad} P_{k}\right\rangle \tilde{V}_{\imath \jmath}
$$

We denote the above vector field (3) by $\bar{X}_{0}$.

LEMMA 3.1. Let $f(x)=P_{k}+P_{k+1}+\cdots$ and $\bar{X}$ and $\bar{X}_{0}$ be as above. Then

$$
\bar{X}_{0}=\operatorname{grad}\left(\left.P_{k}\right|_{S n-1}\right)
$$

where $S^{n-1}$ has the ordinary Riemannian metric.

Remark. The coordinate systems of the both sides of above equation are different, but there will be no confusion.

Proof. Let $T S^{n-1}$ denote the tangent space of $S^{n-1}$. From (2) we easily see that the $T S^{n-1}$ component of $\left.\left(\operatorname{grad} P_{k}\right)\right|_{S_{n-1}}$ is given by (3).

Let $\partial / \partial t$ denote the unit vector on $R$. Then for any vector $v \in T S^{n-1}$, we have

$$
\begin{aligned}
\left\langle\bar{X}_{0}, v\right\rangle \frac{\partial}{\partial t} & =\left\langle T S^{n-1} \text { component of }\left.\left(\operatorname{grad} P_{k}\right)\right|_{s n-1}, v\right\rangle \frac{\partial}{\partial t} \\
& =\left\langle\operatorname{grad}\left(\left.P_{k}\right|_{s n-1}\right), v\right\rangle \frac{\partial}{\partial t}
\end{aligned}
$$




$$
\begin{aligned}
& =\left(P_{k}\right)_{*} v \\
& =\left(\left.P_{k}\right|_{s n-1}\right)_{*} v .
\end{aligned}
$$

Therefore, we have

$$
\bar{X}_{0}=\operatorname{grad}\left(\left.P_{k}\right|_{s n-1}\right) .
$$

Proof of Theorem. Let $g(t)$ be an integral curve of $\operatorname{grad} f(x)$ such that $\lim _{t \rightarrow+\infty} g(t)=0$ and let $L$ denote the $\omega$-limit set of $\lim _{t \rightarrow+\infty} g(t) /|g(t)|$. Let $\tilde{g}(t)$ be the integral curve of the blowing-up vector field $\bar{X}$ with $\tilde{g}(0)=\Phi^{-1}(g(0))$. Then we easily see that

$$
L \times\{0\}=\text { the } \omega \text {-limit set of } \tilde{g}(t) \text { and } L \times\{0\} \subset S^{n-1} \times\{0\} .
$$

From the elementary general theory of dynamical systems, we easily see that $L \times\{0\}$ is a connected closed set and an invariant set by the flow of $\bar{X}_{0}$.

Now, at the point $x \in S p\left(P_{k}\right) \cap S^{n-1}$ the following holds :

$$
x_{i} \frac{\partial P_{k}}{\partial x_{\jmath}}=x_{j} \frac{\partial P_{k}}{\partial x_{\imath}} \quad \text { for any } i, j=1,2, \cdots, n \text {. }
$$

Thus, the position vector $\vec{x}$ and $\operatorname{grad} P_{k}(x)$ are parallel i.e. $\operatorname{grad} P_{k}(x)$ has no $T S^{n-1}$ components. Therefore $x$ is a singular point of $\left.P_{k}\right|_{S n-1}$ and the condition $\operatorname{dim} S p\left(P_{k}\right) \leqq 1$ means that the singularities of $\left.P_{k}\right|_{S n-1}$ (or equivalently the singularities of $\bar{X}_{0}=\operatorname{grad}\left(\left.P_{k}\right|_{s n-1}\right)$ are finite and isolated. Since $L \times\{0\}$ is a connected set, it is enough to prove that $L \times\{0\}$ does not contain regular points of $\bar{X}_{0}$. Then $L \times\{0\}$ is a one point set and $L$ will give the tangential direction $\lim _{t \rightarrow+\infty} g(t) /|g(t)|$.

Now, we suppose that $L \times\{0\}$ contains a regular point $p_{1}$ of $\bar{X}_{0}$. Let $\tilde{g}_{p_{1}}(t)$ be the integral curve of $\bar{X}_{0}$ with $\tilde{g}_{p_{1}}(0)=p_{1}$. Since $L \times\{0\}$ is invariant by the flow of $\bar{X}_{0}$, we have

$$
\tilde{g}_{p_{1}}((-\infty,+\infty)) \subset L \times\{0\} .
$$

From Proposition 2.3 there exist unique points $\lim _{t \rightarrow-\infty} \tilde{g}_{p_{1}}(t)$ and $\lim _{t \rightarrow+\infty} \tilde{g}_{p_{1}}(t)$, we set $q_{1}=\tilde{g}_{p_{1}}(-\infty)$ and $q_{2}=\tilde{g}_{p_{1}}(+\infty)$. Then $q_{1}, q_{2}$ are singular points of $\left.P_{k}\right|_{s n-1}$. Since $L \times\{0\}$ is a closed set, we have $q_{1}, q_{2} \in L \times\{0\}$.

Now, we take small upper half $n$-disks $D_{q_{1}}^{+}$and $D_{q_{2}}^{+}$centered $q_{1}$ and $q_{2}$ on $S^{n-1} \times R^{+}$such that $D_{q_{1}}^{+} \cap S^{n-1} \times\{0\}$ and $D_{q_{2}}^{+} \cap S^{n-1} \times\{0\}$ contain no singular points of $\bar{X}_{0}$ except $q_{1}, q_{2}$, here $R^{+}$denotes the set of non-negative real numbers. Next we take a flow box $W_{1}$ of $\bar{X}$ on $S^{n-1} \times R^{+}$which contains $\tilde{g}_{p_{1}}(t)$ and joins $D_{q_{1}}^{+}$to $D_{q_{2}}^{+}$(see Fig. 1.).

Since $p_{1} \in L \times\{0\}$, there exist $t_{1}<t_{2}<\cdots<t_{i}<\cdots$ such that

$$
\lim _{i \rightarrow+\infty} t_{\imath}=+\infty, \quad \lim _{i \rightarrow+\infty} \tilde{g}\left(t_{\imath}\right)=p_{1} \quad \text { and } \tilde{g}\left(t_{\imath}\right) \in W_{1} \quad \text { for any } i=1,2, \cdots
$$

Let $u_{\imath}$ be the point of the boundary $\partial D_{q_{2}}^{+}$at which the integral curve $\tilde{g}(t)$ started from $\tilde{g}\left(t_{\imath}\right)$ leaves $D_{q_{2}}^{+}$for the first time. Since $\partial D_{q_{2}}^{+}$is compact, the set $\left\{u_{i}\right\}$ has 
the accumulation points. Let $p_{2}$ be one of them. Then $p_{2} \in S^{n-1} \times\{0\}$ and $p_{2} \in$ $L \times\{0\}$, because if $p_{2} \notin \mathrm{S}^{n-1} \times\{0\}$ then the integral curve $g(t)$ arrives at two different points 0 and $\Phi\left(p_{2}\right)$ but this contradicts Lojasiewicz's theorem.

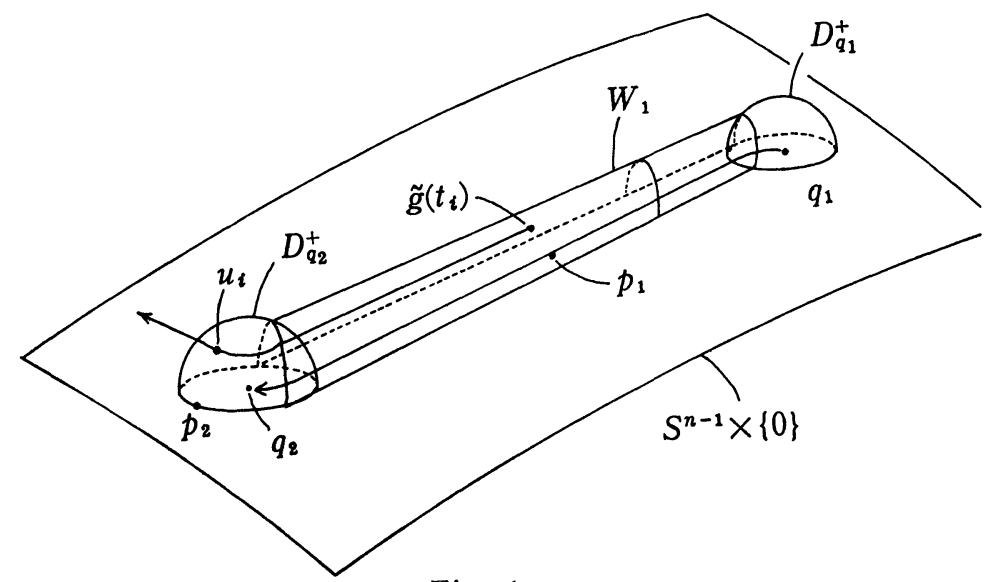

Fig. 1.

Obviously

$$
p_{2} \in L \times\{0\}, \quad \bar{X}_{0}\left(p_{2}\right) \neq 0 \quad \text { and } \quad \tilde{g}_{p_{2}}((-\infty,+\infty)) \subset L \times\{0\} .
$$

We set $q=\lim _{t \rightarrow-\infty} \tilde{g}_{p_{2}}(t)$ and $q_{3}=\lim _{t \rightarrow+\infty} \tilde{g}_{p_{2}}(t)$, then we see that $q=q_{2}$. Because if $q \neq q_{2}$, we take the upper small half $n$-disks $D_{q}^{+}, D_{q_{3}}^{+}$and the flow box $W_{2}$ in the same way as $D_{q_{1}}^{+}, D_{q_{2}}^{+}$and $W_{1}$ with $D_{q}^{+} \cap D_{q_{2}}^{+}=\emptyset, p_{2} \in W_{2}$ and $W_{1} \cap W_{2}=\emptyset$ (see Fig. 2.). Since $u_{i} \in W_{2}$, the integral curve $\tilde{g}(t)$ which starts from $\tilde{g}\left(t_{\imath}\right)$ must pass through a point of $\partial D_{q_{2}}^{+}$different from $u_{\imath}$ and must go into $D_{q}^{+}$and $W_{2}$ before it arrives at $u_{\imath}$. But this contradicts the definition of $u_{\imath}$.

Now, we have the arc $\widetilde{q_{1} q_{2} q_{3}}$ which consists of integral curves $\tilde{g}_{p_{1}}(t)$ and $\tilde{g}_{p_{2}}(t)$ of $\bar{X}_{0}$. If $\widetilde{q_{1} q_{2} q_{3}}$ does not contain a loop, then we repeat the above argument for $D_{q_{3}}^{+}$and costruct the arc $\widetilde{q_{1} q_{2} q_{3} q_{4}}$ and so on. Since the singular points set of $\bar{X}_{0}$ is finite, by the above finite constructions we have the $\operatorname{arc} \widetilde{q_{1} q_{2} \cdots q_{s}}$ which contain a loop $\gamma$. However, $\bar{X}_{0}$ is the gradient vector field of $\left.P_{k}\right|_{s n-1}$, thus the function $\left.P_{k}\right|_{s n-1}$ increases along the integral curve of $\bar{X}_{0}$. This contradics the existance of loop $\gamma$. This completes the proof of Theorem.

LEMMA 3.2. Let $P_{k}(x)$ be a non-zero homogeneous polynomiol of degree $k$ on $R^{n}$. If $\operatorname{dim} S_{p}\left(P_{k}\right)=n$, then $k$ is even and $P_{k}(x)=\alpha r^{k}$ where $\alpha \in \boldsymbol{R}$ and $r=$ $\sqrt{x_{1}^{2}+\cdots+x_{n}^{2}}$.

Proof. In the proof of theorem we see that $S_{p}\left(P_{k}\right) \cap S^{n-1}$ is the singular point set of $\left.P_{k}\right|_{s n-1}$. Since $\operatorname{dim} S_{p}\left(P_{k}\right)=n$, we have $\operatorname{dim} S_{p}\left(P_{k}\right) \cap S^{n-1}=n-1$ i.e. $\left.P_{k}\right|_{s n-1}$ is a constant function. If $k$ is odd then $P_{k}(-x)=-P_{k}(x)$. Thus $\left.P_{k}\right|_{s n-1}$ $\equiv 0$ but this contradicts that $P_{k}(x)$ is non-zero. Hence $k$ is even. We set $\alpha \equiv$ 


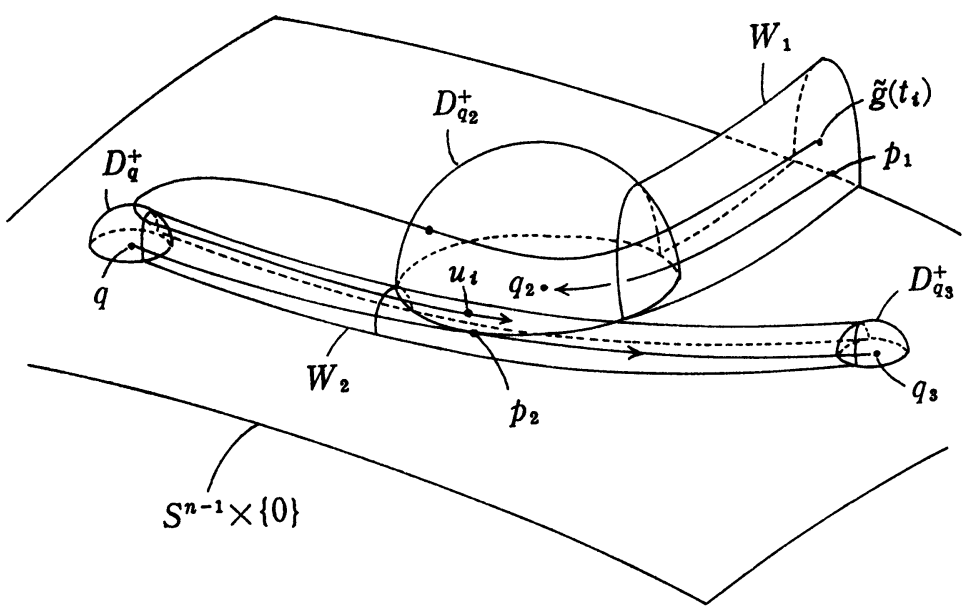

Fig. 2.

$\left.P_{k}\right|_{s n-1}$. Then we have that for any $x \in R^{n}$

$$
P_{k}(x)=P_{k}\left(|x| \frac{x}{|x|}\right)=|x|{ }^{k} P_{k}\left(\frac{x}{|x|}\right)=\alpha r^{k}
$$

PROPOSITION 3.3. Let $f(x)=P_{k}(x)+P_{k+1}(x)+\cdots$ be a real analytic function germ at $0 \in R^{n}$. If $\operatorname{dim} S_{p}\left(P_{k}\right)=n$, then any integral curve $g(t)$ of $\operatorname{grad} f(x)$ which tends to $0 \in R^{n}$ has a unique tangential direction at the origin.

Proof. Let $\bar{X}$ and $\bar{X}_{0}$ be as above. Then, $\bar{X}_{0}$ is given by (3). From Lemma 3.2 we see that $\operatorname{grad} P_{k}=k \alpha r^{k-2} \sum_{i=1}^{n} x_{i} \partial / \partial x_{\imath}$ and we have $\bar{X}_{0} \equiv 0$. Set $\overline{\bar{X}}=(1 / r) \bar{X}$. Then $\bar{X}$ is also a $C^{\infty}$ vector field on $S^{n-1} \times R$ and $\overline{\bar{X}}$ no singular points on $S^{n-1} \times\{0\}$. For $x=g(0)$ the integral curve of $\overline{\bar{X}}$ started from $\Phi^{-1}(x)$ meets $S^{n-1} \times\{0\}$ at a unique point of $S^{n-1} \times\{0\}$. This point gives the tangential direction $\lim _{t \rightarrow+\infty} g(t) /|g(t)|$.

From Theorem and Proposition 3.3 we obtain Corollary.

\section{REFERENCES}

[1] S. Lojasiewicz, Ensembles semi-analytiques, I.H.E.S. Lecture Notes (1965).

[2] F. TAKens, Singularities of vector fields, Publ. Math. I. H.E.S. 43 (1973), 47-100.

[3] R. Tном, Gradients des fonctions analytiques, preprint (1986).

Liberal Arts and Science,

TEIKYO UNIVERSITY OF TECHNOLOGY, 2289, Uruido, Ichihara, Chiba 290-01 JAPAN 